\title{
Through the Looking Glass: Chronic Urticaria Treated with Anti-IgE Therapy
}

\author{
Giovanni B. Pajno, MD
}

\section{Introduction}

Chronic URTICARIa (CU) is a skin condition characterized by the presence of intensively pruritic lesions occurring intermittently or continuously for more than 6 weeks. Because the cause(s) of CU are often not known, it is usually referred as chronic spontaneous urticaria (CSU) or chronic idiopathic urticaria (CIU). It affects $0.1 \%-0.3 \%$ of children. ${ }^{1}$ Moreover, solar urticaria (SU) is a rare chronic photodermatosis that is burdened by frequent reductions in patients' quality of life. ${ }^{2}$ Some individuals with various forms of CU are insufficiently controlled by standard treatment, including high dose of H1-antihistamines. In this issue, Olszowiec-Chlebna et al. report the case of an adolescent female with CSU and idiopathic anaphylaxis who was effectively treated with omalizumab. ${ }^{3}$ Interestingly during the treatment (6 months) and 3 months later, the patient has not presented CSU or anaphylaxis symptoms. Up to now, there is not a single randomized trial on children with urticaria or idiopathic anaphylaxis. Therefore, this case represents a potential strategy to identify an effective treatment for children and adolescents with uncontrolled severe allergic cutaneous disorders.

\section{The Clinical Scenario}

The patient had recurrent episodes of urticaria and angioedema. Therefore, she was treated with corticosteroids and antihistamines. The dose of H1-antihistamines was increased up to 4 times the standard dosage. This dose caused a partial improvement of symptoms. However, when the treatment was interrupted, a relapse appeared within days after cessation of treatment. Similarly to other cases of $\mathrm{CU}$, in this case the etiology of hives was not known. In addition, in following stage the patient was admitted to an emergency department because of anaphylaxis. She was treated with epinephrine, corticosteroids, and inhaled beta- 2 agonists. Self-injectable epinephrine was prescribed and high dose of H1-antihistamines was sustained. The relapse of hives and pruritus despite regular treatment was quite frequent. Therefore, the physicians elected to change her management and administered omalizumab sub- cutaneously $300 \mathrm{mg}$ every 4 weeks. Within few weeks her hives ceased to occur.

\section{No longer waiting time for promising new approaches?}

In refractory patients who have failed traditional first-/ second-line therapies, consideration of omalizumab, and future anti-IgE antibody biologic drugs, may represent effective options. A recent study did show long-term efficacy for omalizumab in adult patients with $\mathrm{CU}^{4}{ }^{4}$ Altogether, the results of childhood case series are encouraging. ${ }^{5}$ However, randomized controlled trials are necessary to identify both effective treatment schedule(s) and dosing for eligible patients suffering from various forms of CU unresponsive to conventional treatment. Safety profiles of omalizumab have been well described in the literature, based on use in patients with severe allergic asthma. ${ }^{6}$ In a study population with CU, 1 patient discontinued omalizumab because of severe headache. Aside from this event, no others adverse reactions were noted in the 19 study subjects. ${ }^{7}$ The authors have demonstrated that omalizumab was effective in the children in this series with CU. However, future studies using a standard protocol and long-term treatment inclusive of multiple CU phenotypes will be required to determine whether long lasting remission can occur and to identify the optimal dosage and duration to achieve it. Improving our understanding of the activity of omalizumab in the inflammatory process associated with various forms of CU: CIS/CSU/SU could also help to inform the decision regarding optimization of omalizumab therapy.

\section{Conclusions}

CU still poses problems with respect to reduced quality of life, lack of efficacy, and expense of current treatments for severe intractable symptoms. This case report provides evidence that the approach with fully humanized recombinant anti-IgE antibody is both feasible and acceptable in a clinical setting and therapeutically powerful for well-selected patients. Other interesting, preliminary data are the usage of omalizumab for prevention and therapy in subjects with CSU and concomitant

Department of Pediatrics, Allergy Unit, University of Messina, Messina, Italy.

(C) Giovanni B. Pajno 2016; Published by Mary Ann Liebert, Inc. This Open Access article is distributed under the terms of the Creative Commons Attribution Noncommercial License (http://creativecommons.org/licenses/by-nc/4.0/) which permits any noncommercial use, distribution, and reproduction in any medium, provided the original author(s) and the source are credited. 
idiopathic anaphylaxis. This is not a matter because omalizumab represents the latest development in the treatment of chronic uncontrolled urticaria. Therefore, omalizumab offers new possibilities to patients with chronic severe symptoms. School-aged children and teenagers with chronic forms of urticaria could be an appropriate target for this therapy.

\section{Author Disclosure Statement}

No competing financial interests exist.

\section{References}

1. Sahiner UM, Civelek E, Tuncer A, et al. Chronic urticaria: Etiology and natural course in children. Int Arch Allergy Immunol 2011; 156:224-230.

2. Zuberbier T, Aberer W, Asero R, et al. The EAACI/GA(2) LEN/EDF/WAO Guideline for the definition, classification, diagnosis and management of urticaria: The 2013 revision ad update. Allergy 2014; 69:868-887.

3. Olszowiec-Chlebna M, Sztafinska A, Stelmach I. Teenager suffered from idiopathic anaphylaxis and chronic spontaneous urticaria successfully treated with omalizumab-A case report. Pediatr Allergy Immunol Pneumol 2016; [Epub ahead of print]; DOI: 10.1089/ped.2015.0580.
4. Kapkan A, Ferrer M, Bernstein JA, et al. Timing and duration of omalizumab response in patients with chronic idiopathic/ spontaneous urticaria. J Allergy Clin Immunol 2016; 137: 474-481.

5. Wahn U. Anti-IgE for chronic urticaria - Are children little adults after all? Pediatr Allergy Immunol 2015; 26:488-489.

6. Chipps BE, Figliomeni M, Spector S. Omalizumab: An update on efficacy and safety in moderate-to-severe allergic asthma. Allergy Asthma Proc 2012; 33:377-385.

7. Viswanathan RV, Moss MH, Mathur SK. Retrospective analysis of the efficacy of omalizumab in chronic refractory urticaria. Allergy Asthma Proc 2013; 34:446-452.

Address correspondence to:

Giovanni B. Pajno, MD

Department of Pediatrics, Allergy Unit

University of Messina

Messina, 98124

Italy

E-mail: giovanni.pajno@unime.it

Received for publication January 9, 2016; accepted after revision January 18, 2016. 